\title{
Product environmental footprint—breakthrough or breakdown for policy implementation of life cycle assessment?
}

\author{
Matthias Finkbeiner
}

Received: 27 November 2013 / Accepted: 3 December 2013 / Published online: 17 December 2013

(C) Springer-Verlag Berlin Heidelberg 2013

Did you hear about the latest footprint? It is not anymore about carbon footprinting (Finkbeiner 2009) or water footprinting (Kounina et al. 2013; Berger and Finkbeiner 2010); we now have to deal with environmental footprints. The EU Commission published the Product Environmental Footprint (PEF) and Organisation Environmental Footprint (OEF) methods (EU 2013a) as part of the Communication "Building the Single Market for Green Products" (EU 2013b). According to the documents released, the PEF and OEF methodologies build on existing life cycle assessment (LCA)-based methods and aim at harmonizing them. They purportedly aim at increasing comparability between products by predefining requirements for certain methodological aspects, thus decreasing the flexibility provided by ISO 14044 (2006). PEF and OEF were developed by the European Commission's Joint Research Centre (JRC). But is PEF really the breakthrough for policy implementation of LCA? Close analysis of the PEF method reveals key concerns from the perspective of state-ofthe-art LCA practice and science. Rather than proposing a harmonized compromise of existing standards, it presents an entirely new one which is even in conflict with the existing ISO 14044 (2006). As such, PEF does not contribute to harmonization, but rather to confusion, proliferation, and mistrust.

M. Finkbeiner $(\bowtie)$

Department of Environmental Technology, Sustainable Engineering,

Technische Universität Berlin, Office Z1, Strasse des 17. Juni 135, 10623 Berlin, Germany

e-mail: matthias.finkbeiner@tu-berlin.de

\section{Do we really need another footprint?}

Carbon footprinting definitely helped the growth and mainstreaming of life cycle-based environmental assessments (Finkbeiner 2009). Water footprinting helped to raise life cycle awareness of the most severe environmental issue in many parts of the world and provided balance to the monophthalmia of carbon footprinting (Kounina et al. 2013; Berger and Finkbeiner 2010). The Ecological Footprint (Wackernagel and Rees 1996) taught us the bitter lesson that media-compatible communication features can be more relevant than scientific substance. But do we really need more footprints? Whatever footprints?

Well, the Product (PEF) and Organisation (OEF) Environmental Footprint might still make a difference because they were published by the EU Commission. As such, they come with a tempting promise: real and substantial policy implementation of LCA. We in the LCA community are eager to get the good things that we have been doing, and still do, finally applied, finally relevant in public and private decision making. Now and then, we experience such real-world relevance in our work for companies, but real, substantial, and meaningful policy use of LCA is unfortunately still rare. Will PEF and OEF lead us to such a promised land in which LCA is properly used in policy making?

The following objectives and features are claimed for the PEF/OEF (EU 2013a, b, c).

- Inclusion of life cycle assessment in environmental policy

- Harmonization of methods to avoid proliferation

- Building on existing, extensively tested, and used methods

- International cooperation 
- Comparability over flexibility

- Cost reduction for business and increased applicability for SMEs

- Credible communication to consumers avoiding confusion and mistrust

The proposal of further guidance for a harmonized method for quantifying and communicating environmental performance from cradle-to-grave is good and supported, but it is also a big challenge. The main objectives and features of PEF/ $\mathrm{OEF}$ as indicated above are not controversial either. However, it is currently unclear whether the proposed approach actually supports these targets in a substantial way or whether the proposal has even an adverse effect on the policy targets it tries to achieve.

We summarize here some key findings of a comprehensive analysis of the PEF/OEF methodology with regard to the consistency with international state-of-the-art LCA standardization (Finkbeiner 2013) and science. The analysis focused in general on the product application (PEF). However, many of the identified issues apply for OEF as well and selected aspects with particular relevance for OEF are also addressed.

\section{Analysis}

As a starting point and one of the main targets, the proposed PEF methodology is supposed to harmonize several existing standards and methods, e.g., ISO 14044 (2006), ISO/TS 14067 (2013); ISO 14025 (2006), ISO 14020 (2000); GHG Protocol (2013a, b), BPX 30-323-0 (AFNOR 2011), and PAS 2050 (2011). This claim implies that there are significant differences between these standards and methods and that $\mathrm{PEF} / \mathrm{OEF}$ will establish a harmonized compromise between them. However, as a matter of fact, ISO 14040 (2006) and ISO 14044 (2006) are the leading basis for all the previous quantification standards; they represent the constitution of LCA. Many of the other standards contain significant amounts of ISO 14040/44 content. With exception of the limited focus of the carbon footprint standards on climate change, all of them reference ISO 14044 as their basis and basically comply with it. On the contrary, the proposed PEF/OEF methodology is in severe conflict with several requirements of ISO 14044 and ISO 14025. The proposed PEF/OEF methodology does not support harmonization because it is not based on ISO 14044 as a solid internationally agreed reference and because the proposed method is more different than any other existing standard. It is not a harmonized compromise of existing standards, it is a new one. As such, it does not contribute to harmonization, but to proliferation.

The following examples show some deviations from globally, basically unanimously agreed harmonized standards:
- A completely new set of terminology is introduced (e.g., environmental footprint instead of life cycle assessment, unit of analysis instead of functional unit, resource use and emissions profile instead of life cycle inventory, and so on). Renaming these common terms has no added value and rather leads to confusion and proliferation.

- Comparative assertions based on weighting are an optional step for PEF studies. If weighting is applied, the methods and results shall be reported under "additional environmental information." It has been a longestablished global consensus (formulated in ISO 14044), that subjective weighting is not to be used for comparative assertions to be disclosed to the public. None of the other standards indicated above challenged this paradigm. However, PEF leaves this harmonized solution and allows weighting and comparative assertions. The proposal to report it under "additional environmental information" is in full and obvious conflict with ISO 14025 because this is explicitly excluded there.

- With regard to OEF, the current draft of ISO/PDTS 14072 (2013) on LCA for organizations clearly states that LCA studies for organizations shall not be used for comparative assertions intended to be disclosed to the public. Again, the OEF approach allows such an application which is in obvious conflict with the global standards.

- Several reporting requirements based on ISO 14044 are not met, e.g., the requirement of a statement that a life cycle impact assessment (LCIA) shall not provide the sole basis of comparative assertion intended to be disclosed to the public of overall environmental superiority or equivalence, as additional information will be necessary to overcome some of the inherent limitations in the LCIA.

It should be noted, that some of these main differences and conflicts of $\mathrm{PEF} / \mathrm{OEF}$ with existing standards are not properly reflected in the comparison of standards provided in Annex X of EU's PEF document (EU 2013a).

As another feature, the proposed PEF/OEF methodology is supposed to build on existing, extensively tested, and used methods. This is a feasible strategy for harmonization and also for cost-effective implementation. In those parts of the PEF/OEF methodology, which are actually taken over from existing standards, the solid and tested ground of the method is achieved. However, in those parts of the methodology, which are newly required by PEF/OEF, the claim to use extensively tested and used methods is no longer true. Elements like the screening step, the ban of cut-off criteria, the end of life-recycling formula, the reviewer qualification scheme, and several of the proposed impact categories and indicators are neither extensively tested nor extensively used. Some of them were never used in real-world decision making in either private or public organizations. 
In 2011, the JRC published a set of recommended life cycle impact assessment methods (ILCD 2011) based on a comprehensive analysis. This set included 14 impact assessment methods on midpoint level and three methods on endpoint level, which are ranked from level I-III, evaluating the maturity of the method. The recommended methods on midpoint level were incorporated into the PEF method without properly reflecting the maturity levels identified. Only two methods (IPCC method for climate change and WMO method for ozone depletion) are characterized with level I ("recommended and satisfactory") and fulfill all the necessary requirements. According to the analysis from the JRC, the methods for assessing water consumption and land use should "only be applied with caution" due to shortcomings of the models and factors (level III). Seven methods are classified as level II ("recommended, some improvements needed"). Finally, all the three USEtox models (human toxicity for cancer and non-cancer effects and ecotoxicity) are ranked as level IIIII. Even the developers of USETox themselves acknowledge that the uncertainty of the characterization factors is up to three orders of magnitude (Rosenbaum et al. 2008) and scientific consensus should not be interpreted as reduced uncertainty (Huijbregts 2013). It is striking that the $\mathrm{PEF} / \mathrm{OEF}$ methodology requires the use of the even most uncertain methods.

Some examples of the most obvious artifacts of the untested methods proposed include the following:

- For assessing impacts of water use, the ecological scarcity method (Frischknecht et al. 2008) is recommended. However, this method contains severe flaws. For instance, impacts resulting from water consumption in Germany (910 eco points $/ \mathrm{m}^{3}$ ) are considered 40 times higher than in Australia (23 eco points $/ \mathrm{m}^{3}$ ). It is a no-brainer to find such deficits in simple user tests, which obviously were not performed. More commonly used and better tested impact assessments for water do not show these artifacts.

- The proposed recycling allocation formula is not used in practice by anyone and therefore not tested. It can lead to unrealistic results since for example the landfill term is never zero even if $100 \%$ of the material is recycled. The quality term can be "adjusted" to almost any "desired" result. The very environmentally relevant aspect of how often a material can be recycled is not reflected in the formula at all. A material that is recycled barely once gets the same burden/credit as a material that is recycled 100 times.

- Acidification and eutrophication impacts are assessed in almost every LCA today, based on tested methods. The PEF proposes the accumulated exceedance method (Seppälä et al. 2006; Posch et al. 2008) which was basically never used and tested. It is better in theory to assess impacts on a regional level but practically impossible as no regional inventory data are available in common LCA datasets. As a consequence, average characterization factors based on European data are provided by the JRC which include overall only five characterization factors for acidification and six for eutrophication. Hence, characterization factors for many inventory flows are missing, and the method is less robust than well-established impact indicators. Due to the limited use of the method, updating and improving it seems difficult as there is currently no maintenance by any organization.

In real-world applications of LCA, there are many existing, extensively tested, and used methods available. Harmonization could have been achieved by selecting among these established methods rather than proposing new, unused, and unproven methodologies.

Under the justification "comparability over flexibility," the PEF/OEF approach predetermines a number of value choices. One feature that is supposed to support "comparability" is the requirement that each "unit of analysis" has to be linked to the CPA/NACE codes (Classification of Products by Activity/Nomenclature statistique des activités économiques dans la Communauté européenne). Product Environmental Footprint Category Rules (PEFCRs) are supposed to be developed on the two-digit level of the classification. However, for many products, the identification of a suitable code is not a straightforward task and many groups cluster products together with very different productions systems (e.g., manufacture of vegetable and animal oils and fats).

The PEF methodology also does not allow cutoff criteria. How this methodological requirement of PEF could practically be followed is completely unclear as it is theoretically impossible to achieve. To include all processes and all elementary flows for the 14 impact categories proposed leads to an exploding workload for identification of flows and related processes with a limited significance for the results (if the cutoff is done properly, the influence on the results is usually negligible).

Regarding data, the PEF methodology provides an evaluation scheme to assess the quality of the used data. Even though this scheme is formally easy to apply, it leaves a lot of room for adjusting the scoring and interpretation. The data quality of the data quality assessment will often be worse than the data quality of the LCA study. It also has to be applied on a case-by-case basis as the evaluation depends on the context of the study. For product systems with easily 100 or more unit processes, the extra unproductive workload is substantial even if only minutes are assumed for each data set. Despite the efforts generated, the required data quality assessment does not allow any quantitative assessment on the significance of the results. 


\section{Matching with policy targets: breakthrough or breakdown?}

The analysis presented above addresses some key concerns from the perspective of state-of-the-art LCA practice and science. In this section, the coherence, efficiency, and effectiveness of the PEF/OEF methodology with regard to the intended policy targets are evaluated.

\section{- Harmonization of methods to avoid proliferation}

PEF and OEF are in conflict with the leading international standards for LCA and EPDs. The proposed PEF/OEF methodology contains more differences and conflicts to ISO 14044 and ISO 14025 than any of the other standards which they claim to harmonize. They do not present a compromise between or further guidance to existing standards; they propose new ones. Harmonization is necessary based on the solid foundation of the existing international standards, but $\mathrm{PEF} /$ OEF does not even conform to the existing harmonization level. As a matter of fact, PEF/OEF does not contribute to more harmonization, but to discord and proliferation.

- Building on existing, extensively tested, and used methods

Several of the newly proposed methodologies have never been used or tested in real-world applications. Some of the untested impact assessment methods were even characterized by the JRC itself as to be "handled with care." They are simply not applicable. They contain severe flaws of scientific relevance like higher water scarcity in Germany than in Australia or Spain. The proposal of such unproven methodologies leads to lacking robustness of results and poses even a threat to the credibility of the LCA method as such.

\section{- International cooperation}

International cooperation is supposed to be supported by PEF. This is of great importance because many environmental interventions of EU consumption actually occur in environments beyond the borders of Europe. However, $\mathrm{PEF} / \mathrm{OEF}$ is a threat for international cooperation because they do not respect unanimously agreed international LCA standards. Many developing countries including China and India raised concerns with footprinting standards with regard to nontariff barriers of trade. It is really important to ensure the proactive contribution of these countries due to their large levers for environmental performance and to keep their support for international standardization (Finkbeiner 2013). PEF/OEF is counterproductive in this respect.

\section{- Comparability over flexibility}

One main justification of several value choices in the PEF/ OEF method is the principle of "comparability over flexibility." First, comparability and flexibility are neither the correct terms for the issue nor the proper antonyms. If there is variability in the real world and a certain modeling approach defines fixed values or a fixed approach for it, that does not increase comparability, it just increases reproducibility. If a certain end of life-recycling approach is fixed, as in PEF/OEF, it increases reproducibility since everyone is forced to use the same formula. However, if this formula represents a best case solution for one material and a worst case solution for another material, it does not introduce comparability, but bias. The same applies to weighting. The PEF/OEF methodology predetermines a limited number of value choices like the selection of impact categories, the end of life recycling, and so on. However, for real reproducibility, the PEF/OEF would need to fix many more methodological choices, most prominently, the data sources and databases. As such, PEF/OEF introduce inflexibility (proper antonym to flexibility), fail to achieve full reproducibility, and do not add at all to the comparability of relevant environmental information.

- Cost reduction for business and increased applicability for SMEs

Cost reduction for business and increased applicability for SMEs are formulated as objectives of the PEF/OEF method. However, there is hardly any feature in the PEF/OEF method that actually supports this target. The requirement to assess numerous highly complex and untested impact categories which result in a need for even regionalized inventory data increases the cost substantially. The requirement of regionalized inventory data is even prohibitive for many applications. The restriction, that cut-offs are not allowed, increases the efforts and reduces the applicability for SMEs substantially. The introduced verification scheme adds cost. The data quality assessment of $\mathrm{PEF} / \mathrm{OEF}$ requires a massive workload since both specific and generic data need to be assessed for every study. If all the requirements for PEF/OEF are followed seriously, the resulting cost for the LCA will easily increase $100 \%$ or more - without any significant increase in LCA quality. As such, $\mathrm{PEF} / \mathrm{OEF}$ is rather a cost driver for business and an obstacle for promoting LCA use with SMEs.

- Credible communication to consumers avoiding confusion and mistrust

Another feature of PEF/OEF is the intention to support credible communication to consumers avoiding confusion and mistrust. Again, the introduction of a new approach with new terminology rather bares the risk of confusion to 
consumers. The proposal of untested and flawed methodology will lead to mistrust as even lay consumers understand thatopposite to what PEF/OEF tries to tell them - water scarcity is worse in Australia and Spain than in Germany. The PEF/OEF methodology also offers very little in the sense of how the communication should actually look like. PEF/OEF provides neither an answer nor a discussion, how they relate to the established environmental labeling schemes like the EU flower or the Blue Angel. The PEF/OEF communication indirectly devalues and challenges the existing product labels and organizational reporting schemes (e.g., environmental statement according to EMAS (EU 2009)), while PEF's and OEF's applicability, let alone superiority, are not proven at all.

- Inclusion of life cycle assessment in environmental policy

$\mathrm{PEF} / \mathrm{OEF}$ chooses the voluntary communication of life cycle environmental performance information to any private, public, and civil society stakeholders as their only policy implementation option. According to the EU Commission's PEF/OEF Recommendation itself, they do not apply to the implementation of EU mandatory legislation that foresees a specific methodology for the calculation of the life cycle environmental performance of products. This restriction casts doubt on the seriousness of the EU Commission's commitment to implementing LCA in environmental policy. The environmental relevance of several pieces of EU legislation - from automotive emissions, via biofuels, substance bans according to RoHS, or ELV to REACH — could be improved substantially if a proper LCA-based policy were introduced. Some of these policy options could be achieved relatively easily and robustly. Contrary to that, consumer communication as the main policy vehicle for LCA is a long, challenging tramp which bears significant risk.

\section{Conclusions and recommendations}

PEF and OEF are supposed to achieve an important goal, the implementation of LCA in European environmental policy. Unfortunately, the proposed methods and solutions do not achieve the objectives which they are supposed to serve. In their current form and due to the significant flaws addressed above, PEF and OEF will not support but rather damage the idea of integrating LCA into environmental policy. Many of the flaws could have been and still could be corrected easilyeven before extensive cost and efforts are put into the planned pilot studies (see the PEF website (EU 2013c) for a list of pilot projects).

The most urgent requirements and improvements for the PEF/OEF method include:

- PEF/OEF needs to commit to conformity with international standards, especially ISO 14040/44. Building harmonization on the solid foundation of ISO 14040/44 may work; harmonization against ISO 14040/44 will lead to proliferation, confusion, and mistrust.

- $\mathrm{PEF} / \mathrm{OEF}$ needs to use internationally agreed terminology. There is no added value at all to inventing new and artificial terminology.

- $\mathrm{PEF} / \mathrm{OEF}$ needs to use internationally agreed and tested impact categories, e.g., all impact categories with a score of I and II only in the ILCD study.

- PEF/OEF needs to use scientifically valid and practically applicable impact category indicators for the selected impact categories, e.g., CML or ReCiPe instead of Seppälä et al. (2006) and Posch et al. (2008) for eutrophication and acidification.

- PEF/OEF needs to refrain from overselling their piecemeal approach to fix a certain set of value choices as a solution for achieving comparability. These "pseudosolutions" (e.g., recycling allocation, weighting, product classification) do not even achieve full reproducibility. PEF/OEF may support comparisons but cannot replace the responsibility of any users to make their own informed choice.

- $\mathrm{PEF} / \mathrm{OEF}$ needs to refrain from adding unrealistic requirements, burden, and cost to LCA application for business and SMEs without adding value, e.g., by the data quality assessment scheme and the restriction of cut-offs.

Is PEF really the breakthrough for policy implementation of LCA? I am far from believing it. I am rather worried that PEF will go down as the gravedigger of substantial policy implementation of LCA in Europe for quite some time. However, hope springs eternal. May the sadly missed sense of (LCA) reality finally find its way into PEF!

\section{References}

AFNOR (2011) General principles for an environmental communication on mass market products. Repository of good practices BP X 30323-0

Berger M, Finkbeiner M (2010) Water footprinting: how to address water use in life cycle assessment? Sustainability 2(4):919-944

EU (2009) Regulation (EC) No 1221/2009 of the European Parliament and of the Council of 25 November 2009 on the voluntary participation by organisations in a Community eco-management and audit scheme (EMAS), Official Journal L 342, 22/12/2009 P. 0001-0045

EU (2013a) Commission Recommendation of 9 April 2013 on the use of common methods to measure and communicate the life cycle environmental performance of products and organisations (2013/179/ EU). Official Journal of the European Union, Volume 56, 4 May 2013

EU (2013b) Building the Single Market for Green Products Facilitating better information on the environmental performance of products and organisations. Communication/* COM/2013/0196 final */ 
EU (2013c) EU Environmental Footprint website. http://ec.europa.eu/ environment/eussd/smgp/product_footprint.htm, accessed 17.11.2013

Finkbeiner M (2009) Carbon footprinting - opportunities and threats. Int J Life Cycle Assess 14(2):91-94

Finkbeiner M (2013) From the 40s to the 70s - the future of LCA in the ISO 14000 family. Int J Life Cycle Assess 18(1):1-4

Frischknecht R, Steiner R, Jungbluth N (2008) The Ecological Scarcity Method-Eco-Factors 2006. A method for impact assessment in LCA. Environmental studies no. 0906. Federal Office for the Environment (FOEN), Bern

GHG Protocol (2013a) Product Life Cycle Accounting and Reporting Standard. http://www.ghgprotocol.org/standards/product-standard, accessed 19.11. 2013

GHG Protocol (2013b) Corporate Value Chain (Scope 3) Accounting and reporting Standard. http://www.ghgprotocol.org/standards/scope-3standard, accessed 19.11. 2013

Huijbregts M (2013) A critical view on scientific consensus building in life cycle impact assessment. Int J Life Cycle Assess. doi:10.1007/ s11367-013-0674-1

ILCD (2011) European Commission-Joint Research Centre - Institute for Environment and Sustainability: International Reference Life Cycle Data System (ILCD) Handbook- Recommendations for Life Cycle Impact Assessment in the European context. First edition November 2011. EUR 24571 EN. Luxemburg. Publications Office of the European Union

ISO 14020 (2000) Environmental labels and declarations - General principles. Geneva, Switzerland

ISO 14025 (2006) Environmental labels and declarations, type IIIenvironmental declarations-principles and procedures. Geneva, Switzerland
ISO 14040 (2006) Environmental management-life cycle assessmentprinciples and framework. Geneva, Switzerland

ISO 14044 (2006) Environmental management-life cycle assessmentrequirements and guidelines. Geneva, Switzerland

ISO/TS 14067 (2013) Carbon footprint of products - requirements and guidelines for quantification and communication. Geneva, Switzerland

ISO/PDTS 14072 (2013) Environmental management—Life cycle assessment-Requirements and guidelines to apply life cycle thinking to organizations. Geneva, Switzerland

Kounina A et al (2013) Review of methods addressing freshwater use in life cycle inventory and impact assessment. Int J Life Cycle Assess 18(3):707-721

PAS 2050 (2011) Specification for the assessment of the life cycle greenhouse gas emissions of goods and services. BSI, London

Posch M, Seppälä J, Hettelingh JP, Johansson M, Margni M, Jolliet O (2008) The role of atmospheric dispersion models and ecosystem sensitivity in the determination of characterisation factors for acidifying and eutrophying emissions in LCIA. Int J Life Cycle Assess $13: 477-486$

Rosenbaum R et al (2008) USEtox - the UNEP-SETAC toxicity model: recommended characterisation factors for human toxicity and freshwater ecotoxicity in life cycle impact assessment. Int J Life Cycle Assess 13:532-546

Seppälä J, Posch M, Johansson M, Hettelingh JP (2006) Countrydependent characterisation factors for acidification and terrestrial eutrophication based on accumulated exceedance as an impact category indicator. Int J Life Cycle Assess 11:403-416

Wackernagel M, Rees W (1996) Our ecological footprint. New Society Publishers, Canada 